\title{
Effect of fibroblast co-culture on the proliferation, viability and drug response of colon cancer cells
}

\author{
BYUMSEOK KOH ${ }^{1}$, HYOJIN JEON ${ }^{2}$, DAHEE KIM $^{1}$, DUKJIN KANG ${ }^{3}$ and KWANG ROK KIM ${ }^{2}$ \\ ${ }^{1}$ Bio Platform Technology Research Center; ${ }^{2}$ Innovative Target Research Center, \\ Korea Research Institute of Chemical Technology, Daejeon 34114; ${ }^{3}$ Center for Bioanalysis, Korea \\ Research Institute of Standards and Science, Daejeon 34113, Republic of Korea
}

Received June 16, 2018; Accepted December 10, 2018

DOI: $10.3892 / \mathrm{ol} .2018 .9836$

\begin{abstract}
Interactions between cancer cells and the surrounding fibroblasts serve an important role in cancer proliferation. Colon cancer co-culture model with colon fibroblasts and two metastatic models with lung and skin fibroblasts were established, and the co-culture effects on colon cancer cell proliferation, apoptosis and drug response were evaluated. Co-culture with CCD-18Co and BJ reduces SW480 cell proliferation by 4.2 and 5.3\%, respectively, while WI-38 acts as a positive regulator and increases SW480 cell proliferation by $36 \%$. CCD-18Co and BJ co-culture can also enhance XAV939 potency against SW480 cells by 16.8 and $27.3 \%$; however, WI-38 co-culture reduces the effect of XAV939 by $38.2 \%$. The present results suggest that, depending on fibroblast type, co-culture can have a positive/negative influence on colon cancer growth; therefore, care should be taken when considering fibroblasts as a target for future cancer therapies.
\end{abstract}

\section{Introduction}

Colon cancer is the fourth most common cancer and is one of the leading causes of cancer-related mortality in the United States (1-5). Numerous therapies have been developed with certain therapies involving direct targeting of the cancer, modulating the immune system or blocking angiogenesis (6-9); however, $>60 \%$ have failed in clinical trials for various reasons $(10,11)$. This high failure rate in clinical trials has caused there to be increasing research attention on the tumor

Correspondence to: Dr Dukjin Kang, Center for Bioanalysis, Korea Research Institute of Standards and Science, 267 Gajeong-ro, Yuseong-gu, Daejeon 34113, Republic of Korea

E-mail: djkang@kriss.re.kr

Dr Kwang Rok Kim, Innovative Target Research Center, Korea Research Institute of Chemical Technology, 141 Gajeong-ro, Yuseong-gu, Daejeon 34114, Republic of Korea

E-mail:kkrok@krict.re.kr

Key words: co-culture, colon cancer, proliferation, drug response, apoptosis microenvironment (TME) and on novel therapies capable of targeting the TME (12-14). Previous studies have demonstrated that colon cancer proliferation is not only dependent on cell intrinsic properties, but is also greatly influenced by the TME $(15,16)$. Colon cancer cells may also modulate the TME to create a more favorable environment for proliferation and invasion $(17,18)$. Therefore, the TME and its components may serve as important factors in colon cancer proliferation $(19,20)$. Fibroblasts, as the building blocks of connective tissues, are key components of the TME (21-23). It has been proposed that cancer-associated fibroblasts (CAFs) aid tumors to grow and are associated with all stages of cancer $(24,25)$. Meanwhile, other research has suggested that normal fibroblasts inhibit cancer cell proliferation and metastasis at the during early-stage cancer (26). While studies into CAFs and their influence on cancer progression have been conducted $(27,28)$, systematic studies on fibroblasts and their co-culture effects on the proliferation and drug response of colon cancer cells are rare. Furthermore, interactions between colon cancer cells and fibroblasts from different tissues are relatively unexplored and may provide insight into the proliferation of early stage or metastatic cancer. To address this unresolved subject, the present study established 2D and anchorage-independent colon cancer cell/fibroblast co-culture systems. Using these, the co-culture effects of fibroblasts on cancer cell proliferation and apoptosis were studied, with particular focus on the positive or negative regulatory role of different fibroblast cell lines on colon cancer growth. In addition, the response of colon cancer cells to the Wnt/ $\beta$-catenin inhibitor XAV939 as well as other anticancer agents, including 5-fluorouracil (5-FU), doxorubicin (DOX), camptothecin (CPT) and irinotecan (IRI), was examined in the presence or absence of fibroblasts to determine the co-culture effects.

\section{Materials and methods}

Cells and reagents. The SW480, HT-29, DLD-1 and Caco-2 colon cancer cell lines were obtained from the American Type Culture Collection (ATCC, Manassas, VA, USA). Lung fibroblasts (WI-38), skin fibroblasts (BJ) and colon fibroblasts (CCD-18Co) were also purchased from the ATCC. Ultrapure water (molecular biology grade), RPMI-1640 medium, fetal bovine serum (FBS), 10x tris-buffered saline and Dulbecco's 
phosphate-buffered saline were purchased from Welgene (Gyeongsan, Korea). RPMI-1640 medium powder, TrypLE Express and penicillin-streptomycin were purchased from Thermo Fisher Scientific, Inc., (Waltham, MA, USA). Agar powder, agar solubilization solution, $8 \mathrm{x}$ lysis buffer and Cyqant ${ }^{\circledR}$ GR dye were purchased from Cell Biolabs, Inc., (San Diego, CA, USA). Cell culture flasks ( 25 and $75 \mathrm{~cm}^{2}$ ), cell culture dishes $(60 \times 15 \mathrm{~mm}), 24$-well clear flat-bottom TC-treated multiwall cell culture plates and 6.5 -mm Transwell ${ }^{\circledR}$ polycarbonate membrane inserts with $0.4-\mu \mathrm{m}$ pores were purchased from Corning (Corning, NY, USA). XAV939, IRI, CPT, DOX and sodium bicarbonate were purchased from Sigma-Aldrich (Merck KGaA, Darmstadt, Germany). 5-FU and a Muse Caspase-3/7 assay kit were also from Merck KGaA. A Cell Counting Kit-8 (CCK-8) was purchased from Dojindo Laboratories (Kumamoto, Japan). Pre-cast gels for western blotting were purchased from Bio-Rad Laboratories, Inc., (Hercules, CA, USA). Amersham ${ }^{\mathrm{TM}}$ ECL $^{\mathrm{TM}}$ Select western blot detection reagent was purchased from GE Healthcare (Chicago, IL, USA).

Colon cancer cell co-culture with fibroblasts. The SW480, HT-29, DLD-1 and Caco-2 colon cancer cells and WI-38, CCD-18Co and BJ fibroblasts were cultured in RPMI-1640 medium supplemented with $10 \%$ FBS and $1 \%$ penicillin-streptomycin at $37^{\circ} \mathrm{C}$ in a $5 \% \mathrm{CO}_{2}$ incubator. For the co-culture, 30,000 SW480, HT-29, DLD-1 and Caco-2 cells were seeded onto 24-well plates with $500 \mu \mathrm{l}$ RPMI-1640 medium. Additionally, 30,000 WI-38, CCD-18Co and BJ cells were seeded onto Transwell ${ }^{\circledR}$ inserts with $100 \mu 1$ RPMI-1640 medium. After $6 \mathrm{~h}$, the fibroblasts seeded onto Transwell ${ }^{\mathrm{B}}$ inserts were transferred to a 24-well plate for co-culturing with colon cancer cells. As a blank control, $100 \mu 1$ RPMI-1640 medium without fibroblasts in Transwell ${ }^{\circledR}$ inserts was prepared. Media were replaced with fresh RPMI-1640 medium every 2 days. Colon cancer cell proliferation on day 5 in the presence or absence of fibroblasts was determined with a CCK-8 assay. Briefly, $50 \mu$ l CCK-8 solution was added to each well and incubated for $2 \mathrm{~h}$, following which the absorbance at $450 \mathrm{~nm}$ was measured using a microplate reader (SpectraMax i3; Molecular Devices, San Jose, CA, USA). The apoptotic rate of co-cultured colon cancer cells was measured with a Muse ${ }^{\circledR}$ cell analyzer (Merck KGaA) using the Muse ${ }^{\circledR}$ Caspase-3/7 Assay kit. Cell images were obtained using an Olympus IX 73 inverted microscope (Tokyo, Japan).

Anchorage-independent colon cancer cell culture. To optimize anchorage-independent cell culture, 200-350 $\mu 1$ of $0.1-2.5 \%$ agar solution was mixed with RPMI-1640 medium containing 5,000-200,000 colon cancer cells, solidified at $4^{\circ} \mathrm{C}$ for 3-30 min in 24-well plates and cancer cell proliferation was assessed. Following optimization, $300 \mu \mathrm{l}$ of $0.6 \%$ agar mixed with RPMI-1640 medium containing 30,000 colon cancer cells was solidified for $20 \mathrm{~min}$ at $4^{\circ} \mathrm{C}$ on top of $200 \mu \mathrm{l}$ of $0.6 \%$ agar containing RPMI-1640 medium. Subsequently, $400 \mu 1$ RPMI-1640 medium was added to each well. For fibroblast co-culture, 30,000 WI-38, CCD-18Co or BJ cells seeded in 24-well Transwell ${ }^{\circledast}$ inserts were placed on top of anchorage-independent cultured colon cancer cells. For quantitation of anchorage-independent colon cancer cell growth, the agar-cell layer was solubilized and fluorescence at $520 \mathrm{~nm}$ was measured following the addition of CyQuant ${ }^{\circledR}$ dye. The apoptotic rates of the anchorage-independent cultured colon cancer cells were measured with the Muse ${ }^{\circledR}$ cell analyzer and the Muse ${ }^{\circledR}$ Caspase-3/7 assay kit.

Colon cancer cell proliferation in fibroblast conditioned medium. Fibroblast conditioned RPMI-1640 medium, after 2 days of incubation with $1 \times 10^{6} \mathrm{WI}-38$, BJ and CCD-18Co cells, was collected and centrifuged twice at $700 \mathrm{x}$ g for $3 \mathrm{~min}$ to remove dead cells/cellular debris. Supernatants were collected and diluted with fresh RPMI-1640 medium and incubated with 2D/anchorage-independent colon cancer cells. Fibroblast conditioned media were replaced every 2 days. After 4 days of colon cancer cell/fibroblast conditioned medium co-culture, cells were collected, and rates of proliferation and apoptosis were measured as above.

Co-culture effect on cancer cell proliferation following anti-cancer drug treatment. A total of 30,000 SW480, HT-29, DLD-1 and Caco-2 cells were seeded/mixed with agar in 24-well plates in the presence/absence of WI-38, BJ or CCD-18Co fibroblasts. High concentrations of anti-cancer drugs were used to induce strong reduction in colon cancer cell viability and reveal the effect of fibroblast co-culture. Specifically, 0.1-100 $\mu \mathrm{M}$ XAV939 was added on day 2 to the co-cultured colon cancer cells. Cell culture medium was replaced with fresh RPMI-1640 medium containing 0.1-100 $\mu \mathrm{M}$ of XAV939 on day 4 and cell proliferation was measured on day 5. For other anti-cancer drugs, $10 \mu \mathrm{M}$ 5-FU, IRI, CPT or DOX was added to the colon cancer cells on day 2, and fresh RPMI-1640 medium containing equal concentrations of 5-FU, IRI, CPT or DOX was added on day 4, and cell proliferation was measured on day 5. DLD-1, HT-29 or Caco-2 cell anti-cancer drug tests were conducted as afore described with SW480 cells.

Statistical analysis. Statistical analysis was performed using Origin (v.8.1; OriginLab, Northampton, MA, USA) and GraphPad Prism (v.6; GraphPad Software, Inc., La Jolla, CA, USA). Each experiment was performed in triplicate and values were expressed as the mean \pm standard deviation. Statistical significance were examined by analysis of variance with Tukey's post hoc test. $\mathrm{P}<0.05$ was considered to indicate a statistically significant difference.

\section{Results}

Effect of fibroblast co-culture on colon cancer cell proliferation. To determine the influence of fibroblast co-culture on colon cancer cell proliferation, a non-contact co-culture system was established. Briefly, SW480, DLD-1, HT-29 or Caco- 2 cells were seeded onto a 24 well plate, and three different fibroblasts (WI-38, BJ or CCD-18Co) were placed on inserts and co-cultured with the colon cancer cells. SW480 cells exhibited a 36\% increase in proliferation rate in the presence of WI-38 cells, while showing little or no changes in proliferation rate in the presence of $\mathrm{BJ}$ or CCD-18Co cells (Fig. 1A and B). WI-38 co-culture decreased SW480 cell apoptosis by $26.3 \%$, while BJ or CCD-18Co co-culture had little or 
A

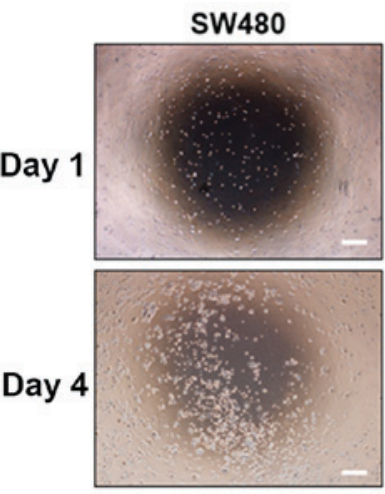

SW480 + WI-38
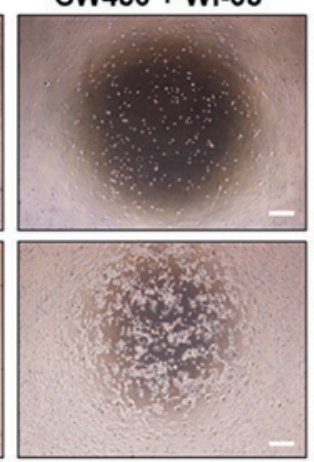

Caco-2
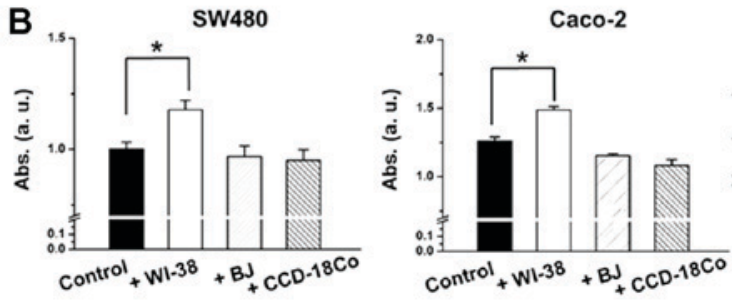

C
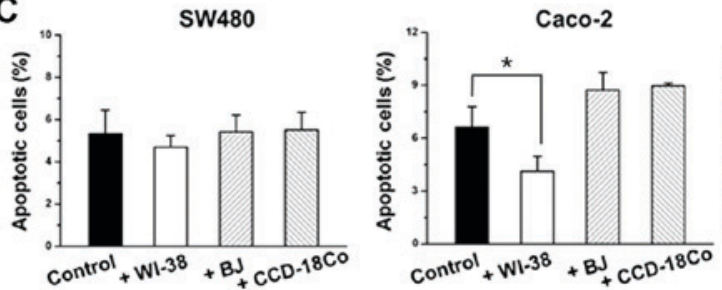
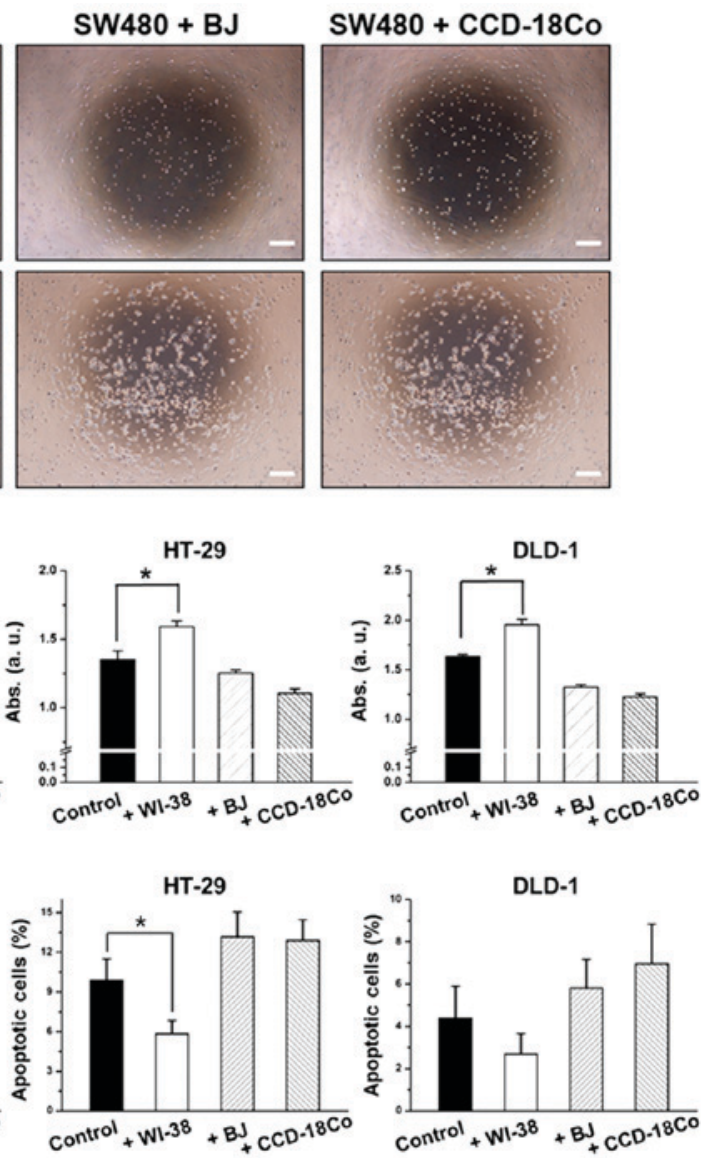

Figure 1. Effect of WI-38, BJ and CCD-18Co cells on colon cancer cells. (A) 2D growth morphology of SW480 cells. (B) Cell proliferation determined by Abs and (C) apoptotic ratio of SW480, Caco-2, HT-29 and DLD-1 cells. Error bars represent the standard deviation of three replicates. ${ }^{*}<0.05$, as indicated. Scale bars, $50 \mu \mathrm{m}$. Abs, absorbance.

no effect, compared with the controls (SW480 cells without fibroblasts; Fig. 1C). Higher proliferation rates for SW480 cells were observed at the center of the well where co-cultured WI-38 cells were closer (Fig. 1A). This was not observed for SW480 cells co-cultured with BJ or CCD-18Co cells. HT-29, DLD-1 and Caco-2 cells co-cultured with fibroblasts exhibited similar results, wherein WI-38 cells induced increases in cell proliferation with increased cell proliferation while BJ and CCD-18Co cells induced decreases in cell proliferation with decreased cell proliferation (Fig. 1B and C).

Fibroblast co-culture and anchorage-independent colon cancer cell growth. To verify the effects of fibroblasts on 3D colon cancer cell growth, an anchorage-independent SW480 cell co-culture was conducted (29). Prior to the fibroblast co-culture, the agar concentration, matrix volume, agar solidifying time and colon cancer cell seeding number was optimized to determine proliferative anchorage-independent cell culture conditions (Fig. 2A-D). The degree of colony formation differed by cell type, and SW480 cells exhibited the highest colony formation efficiency followed by Caco-2, HT-29 and DLD-1 cells (Fig. 2E). Following optimization, colon cancer cells in an agar layer were co-cultured with fibroblasts, and colon cancer cell proliferation, apoptotic and colony formation rates were monitored. Average cell colony diameter increased by $39.4 \%$ (day 6 ) and $56.6 \%$ (day 14) when SW480 cells were co-cultured with WI-38 cells (Fig. 3A and B). By contrast, BJ or CCD-18Co co-culture did not induce a significant change in cell colony diameter (Fig. 3A and B). WI-38 cell co-culture induced a $24 \%$ increase in SW480 cell proliferation, while co-cultures with BJ and CCD-18Co cells decreased the apoptotic rate of SW480 cells by 3.2 and $4.3 \%$, respectively, by day 8 (Fig. 3C). WI-38 and BJ cell co-cultures induced an 84 and $23.3 \%$ increase, while that with CCD-18Co cells induced a $7.8 \%$ decrease, in the SW480 cell colony forming rate (Fig. 3D). WI-38 cell co-culture induced a $22.4 \%$ decrease in the apoptotic rate of anchorage-independent SW480 cells, whereas BJ and CCD-18Co cell co-cultures induced a $2.3 \%$ decrease and a 17\% increase in the SW480 cell apoptotic rate, respectively (Fig. 3E).

Colon cancer cell growth in fibroblast-cultured medium. Proliferation and apoptotic rates of SW480 cells were monitored in the presence of WI-38, BJ or CCD-18Co conditioned medium to determine whether the co-culture effects were mediated by factors released from the fibroblasts. WI-38, $\mathrm{BJ}$ and CCD-18Co conditioned media were mixed (1:3, 1:1 ratio) or unmixed with fresh RPMI-1640 medium and used for 2D/anchorage-independent culture of SW480 cells. Increasing concentrations of WI-38 conditioned medium induced higher rates of proliferation in 2D-cultured SW480 cells $(35.3,49.1$ and $95.3 \%$ increases for $1 / 4,1 / 2$ and full 

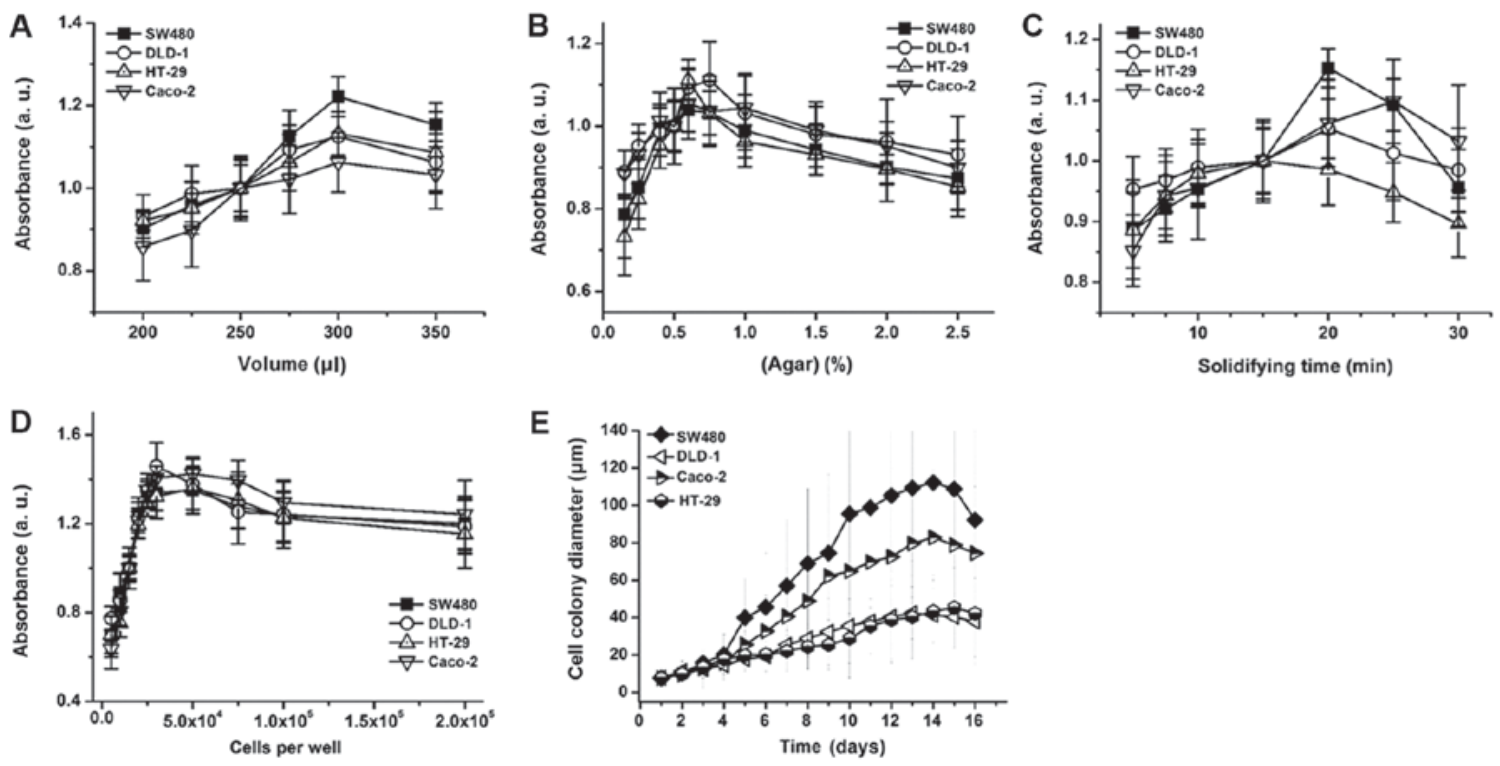

Figure 2. Anchorage-independent colon cancer cell culture optimization. Colon cancer cell proliferation depending on (A) cell matrix volume, (B) matrix agar concentration, (C) matrix solidifying time at $4^{\circ} \mathrm{C}$ and (D) the number of cells (per well/24-well plate). (E) Colon cancer cell diameter changes over time. Error bars represent the standard deviation of three replicates.

WI-38 conditioned medium, respectively; Fig. 4A and B). Proliferation of anchorage-independent SW480 cells also increased by $17.7,24.3$ and $46.2 \%$ in the presence of $1 / 4,1 / 2$ and full WI-38-cultured medium, respectively (Fig. 4B). The apoptotic rates of 2D and anchorage-independent SW480 cells changed by $>5 \%$ in the presence of WI-38 conditioned medium (Fig. 4C). BJ conditioned medium induced a decrease in 2D and anchorage-independent cultured SW480 cell proliferation by 33.8 and $15.8 \%$, respectively, while CCD-18Co conditioned medium reduced proliferation in $2 \mathrm{D}$ and anchorage-independent SW480 cells by 34.3 and $18.4 \%$, respectively (Fig. 5A). BJ and CCD-18Co conditioned media did not induce $>5 \%$ changes in the apoptotic rates of $2 \mathrm{D}$ and anchorage-independent SW480 cells (Fig. 5B).

Fibroblast co-culture effect on colon cancer cell drug responses. Additionally, it was investigated whether fibroblast co-culture could mediate changes in cancer cell responses to anti-cancer drugs. XAV939 exerted low anti-cancer activity against 2D-cultured SW480 cells, causing a $40.5 \%$ decrease in cell proliferation at $100 \mu \mathrm{M}$ (Fig. 6A). BJ and CCD-18Co co-cultures induced 1.1 and $2.1 \%$ increases in XAV939 sensitivity, respectively (Fig. 6A). WI-38 co-culture increased SW480 proliferation by $27.5 \%$ against $100 \mu \mathrm{M}$ XAV939 (Fig. 6A). Anchorage-independent SW480 cells were more sensitive to treatment with XAV939 (Fig. 6B); at $10 \mu \mathrm{M}$, XAV939 reduced the proliferation of anchorage-independent SW480 cells by $29.7 \%$, and $100 \mu \mathrm{M}$ XAV939 decrease SW480 proliferation by $96.1 \%$ (Fig. 6B). BJ and CCD-18Co co-cultures enhanced the anti-cancer activity of $10 \mu \mathrm{M}$ XAV939 by 16.8 and $27.5 \%$, respectively. By contrast, WI-38 co-culture reduced XAV939 activity by $\sim 38.2 \%$ over a $10-50 \mu \mathrm{M}$ range. Additionally, the anti-cancer activity of 5-FU, IRI, CPT and DOX (10 $\mu \mathrm{M}$ single concentrations) was tested against 2D/anchorage-independent SW480 cells. WI-38 co-culture reduced the anti-cancer activity of IRI by $33.8 \%$, and of Dox by
$55.7 \%$, in 2D-cultured SW480 cells, while BJ and CCD-18Co co-culture only exhibited $<10 \%$ activity differences for all 4 drugs (Fig. 6C). For anchorage-independent SW480 culture, WI-38 co-culture reduced anti-cancer activity by 17.4 (5-FU) to 48.6 (DOX)\%, while BJ and CCD-18Co co-cultures enhanced anti-cancer activity by on average 16.2 and $23.5 \%$ in anchorage-independent SW480 cells, respectively (Fig. 6D). The effect of co-culture on the anti-cancer activity of XAV939 against the 3 other initial colon cancer cell lines (HT-29, DLD-1 and Caco-2) was also tested. Similar to its effect with SW480 cells, WI-38 co-culture reduced the anti-cancer activity of XAV939 against HT-29, DLD-1 and Caco-2 cells, while BJ or CCD-18Co co-culture only slightly enhanced the anti-cancer activity (Fig. 7A-F).

\section{Discussion}

Previous studies have shown that the CAF secretome includes angiogenic factors (vascular endothelial growth factor and fibroblast growth factor 1), growth factors [epidermal growth factor (EGF) and transforming growth factor- $\beta$ ] and matrix-modulating factors (matrix metalloproteinases and tissue inhibitors of metalloproteinases), aiding cancer cells to survive, proliferate and become invasive (30-32). Other studies have suggested that normal fibroblasts serve as negative modulators of cancer and inhibit cancer growth and metastasis $(26,33)$. Additionally, decreases in the anti-cancer drug responses of cancer cells co-cultured with fibroblasts have been observed, and factors produced by co-cultured fibroblasts have been shown to promote tumor cell proliferation and resistance against anti-tumor agents $(34,35)$. These findings warranted investigation into whether different types of fibroblasts have different impacts on colon cancer cell proliferation and drug response. In this study we have selected colon fibroblasts (CCD-18Co), lung fibroblasts (WI-38) and skin fibroblasts (BJ) for co-culture with colon cancer cells to mimic the colon 


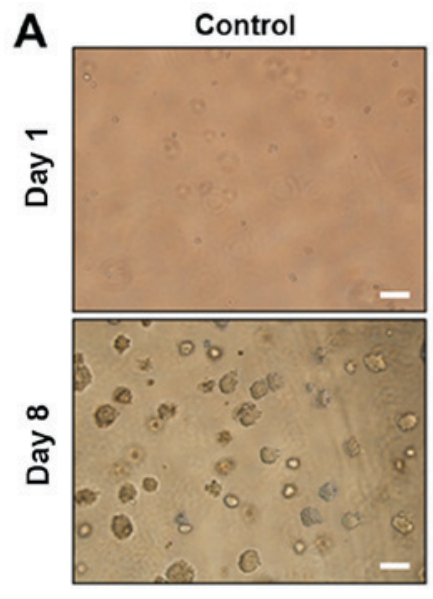

B

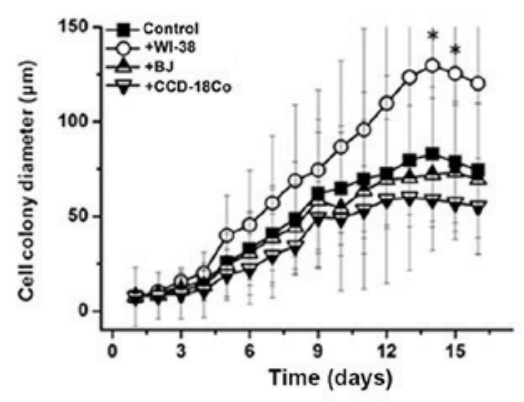

D

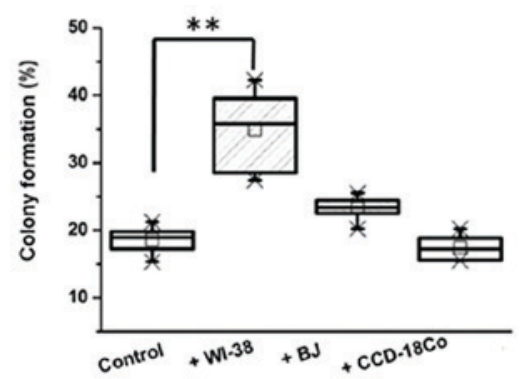

$+\mathrm{BJ}$
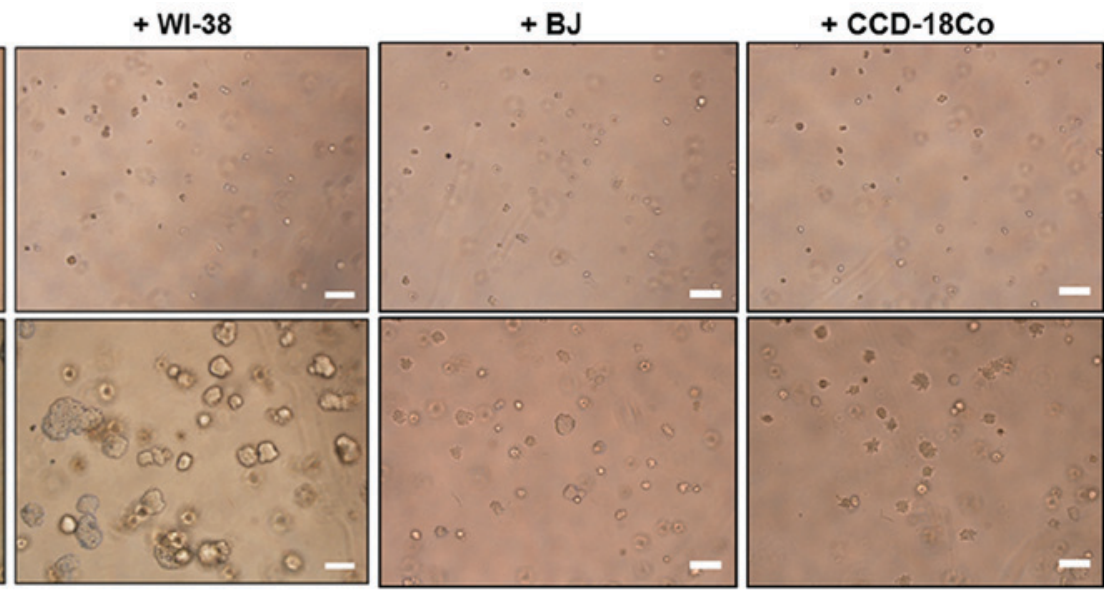

C

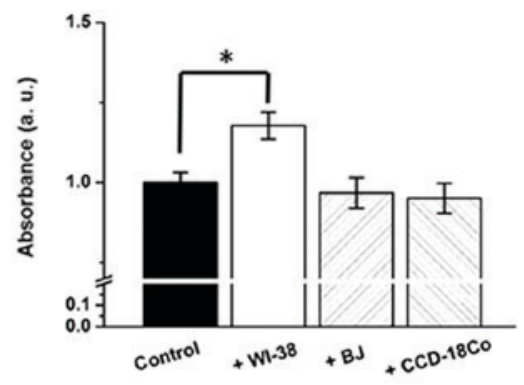

E

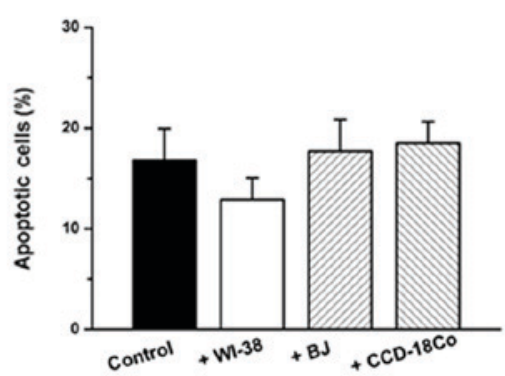

Figure 3. Fibroblast co-culture effect on (A) colony morphology, (B) colony size, (C) cell proliferation, (D) percentage colony formation and (E) apoptotic rate of anchorage-independent SW480 cells. Error bars represent the standard deviation of three replicates. Scale bars, $50 \mu \mathrm{m}$. ${ }^{*} \mathrm{P}<0.05$ and ${ }^{* *} \mathrm{P}<0.01$ vs. control/as indicated.

cancer microenvironment and two highly metastatic sites of colon cancer $(36,37)$. Colon cancer cells with low (Caco-2 and HT-29), medium (SW480) and high (DLD-1) invasiveness $(38,39)$ were selected. A non-contact co-culture system was adopted using an insert so that the cancer cell/fibroblast interactions could be achieved via the sharing of culture medium through micropores. Furthermore, proliferation, apoptosis for each could be successfully determined as the two cell types were physically separated by membranes.

The present data suggested that SW480 cells co-cultured with WI-38 cells were significantly promoted to undergo cell proliferation, with little or no changes in apoptotic rate, compared with controls. To determine whether the co-culture effect was induced by factors released from the fibroblasts, SW480 cells were cultured in WI-38 conditioned medium and the effects on colon cancer cell progression were monitored. SW480 cells exhibited increased proliferation in the presence of the WI-38 conditioned medium, as compared with the controls. This suggests that factors in the WI-38 conditioned medium have a pro-proliferative effect on colon cancer cells. Additionally, BJ and CCD-18Co co-culture induced a decrease in SW480 cell proliferation, but did not induce changes in apoptotic rate. Previous studies have demonstrated that cancer cell proliferation is not only associated with apoptosis but also with various other factors, and that the balance between antiand pro- apoptotic gene expression overall contributes to tumor proliferation and progression $(40,41)$. SW480 cells cultured with BJ or CCD-18Co conditioned medium also exhibited decreased proliferation. This suggests that fibroblast co-culture influences colon cancer cell proliferation is dependent on cell type, and its effects on cancer cells are more complex than simply differentiating between normal fibroblasts and CAFs. Further tests were conducted to investigate whether fibroblast co-culture influences anchorage-independent cultured colon cancer cells. Prior to co-culture, the anchorage-independent culture protocol was optimized to determine the best conditions for colon cancer cell proliferation; this was determined to be sensitive to matrix volume, concentration, solidifying time and the number of cells in each matrix. Colon cancer cells formed multiple colonies after 3 days of anchorage-independent culture. Different degrees of colony forming efficiency were observed between 4 different colon cancer cell types; 
A

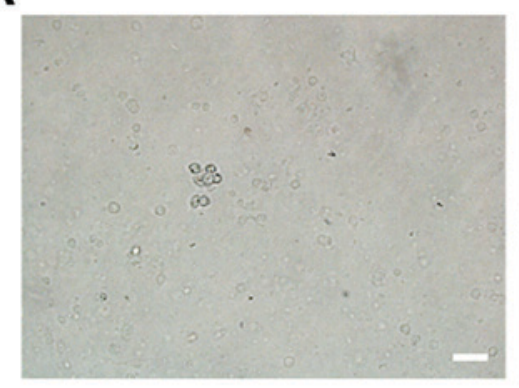

B

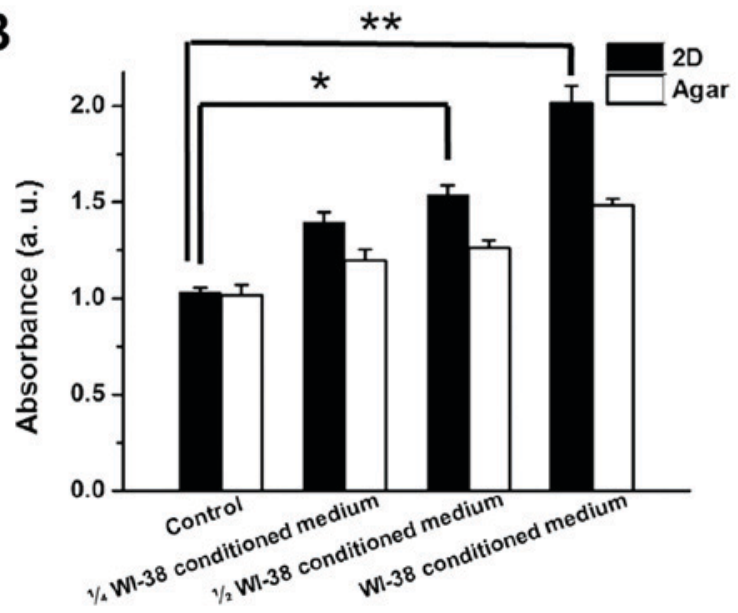

$1 / 2 \mathrm{WI}-38$ conditioned medium

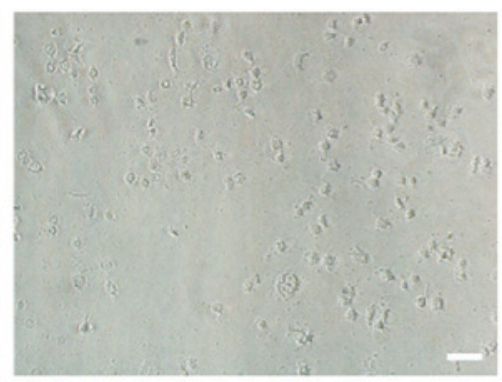

C

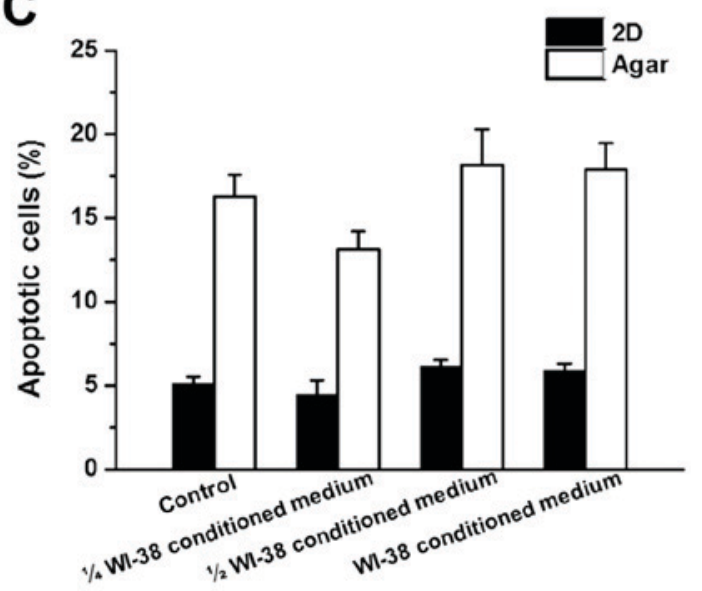

Figure 4. (A) Morphology, (B) growth and (C) apoptotic rate of SW480 cells in the presence of WI-38 conditioned medium. Error bars represent the standard deviation of three replicates. Scale bars, $10 \mu \mathrm{m}$. $\mathrm{P}<0.05$ and ${ }^{* *} \mathrm{P}<0.01$, as indicated.
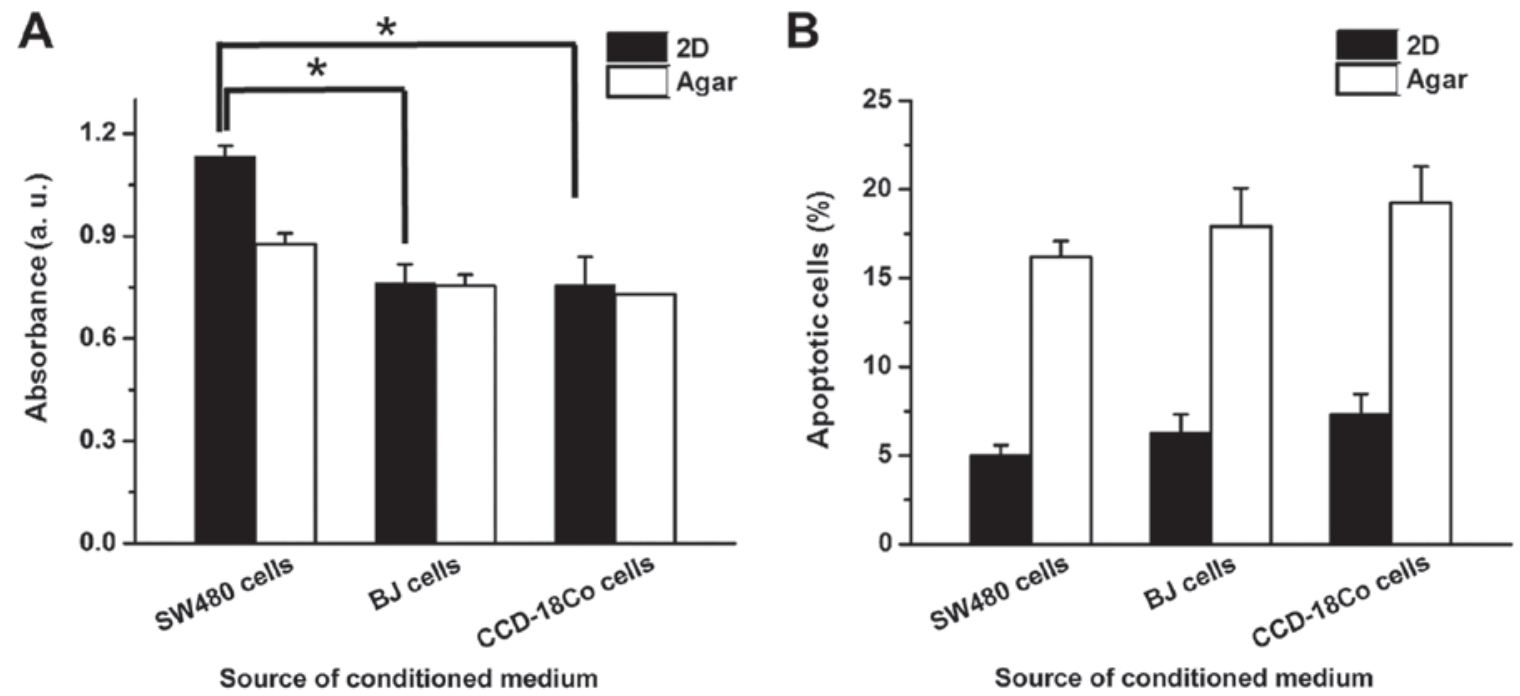

Figure 5. Changes in the (A) proliferation and (B) apoptotic rate of SW480 cells with SW480, BJ or CCD-18Co conditioned medium. Error bars represent the standard deviation of three replicates. ${ }^{*} \mathrm{P}<0.05$, as indicated.

however, correlations between colon cancer cell invasiveness and colony formation ability were not observed using this system. Colon cancer cells that underwent anchorage-independent culture exhibited an average 3.2-fold increase in apoptotic rate when compared with those in $2 \mathrm{D}$ culture. It is speculated that higher apoptotic rate in anchorage-independent culture may be attributed to the hypoxic condition of the system (42).

Following protocol optimization, colon cancer cells in an agar matrix were co-cultured with WI-38, BJ and
CCD-18Co cells. WI-38 cells not only induced an increase in colon cancer cell proliferation, but also induced and increase in colon cancer cell colony size and colony forming rate. $\mathrm{BJ}$ and $\mathrm{CCD}-18 \mathrm{Co}$ fibroblasts decreased colon cancer cell proliferation while having little or no effect on colony forming efficiency. It was further evaluated whether fibroblast co-culture modulated the anti-cancer drug response of colon cancer cells. The Wnt signaling inhibitor XAV939 was selected as a test drug to apply to colon cancer cells, since 

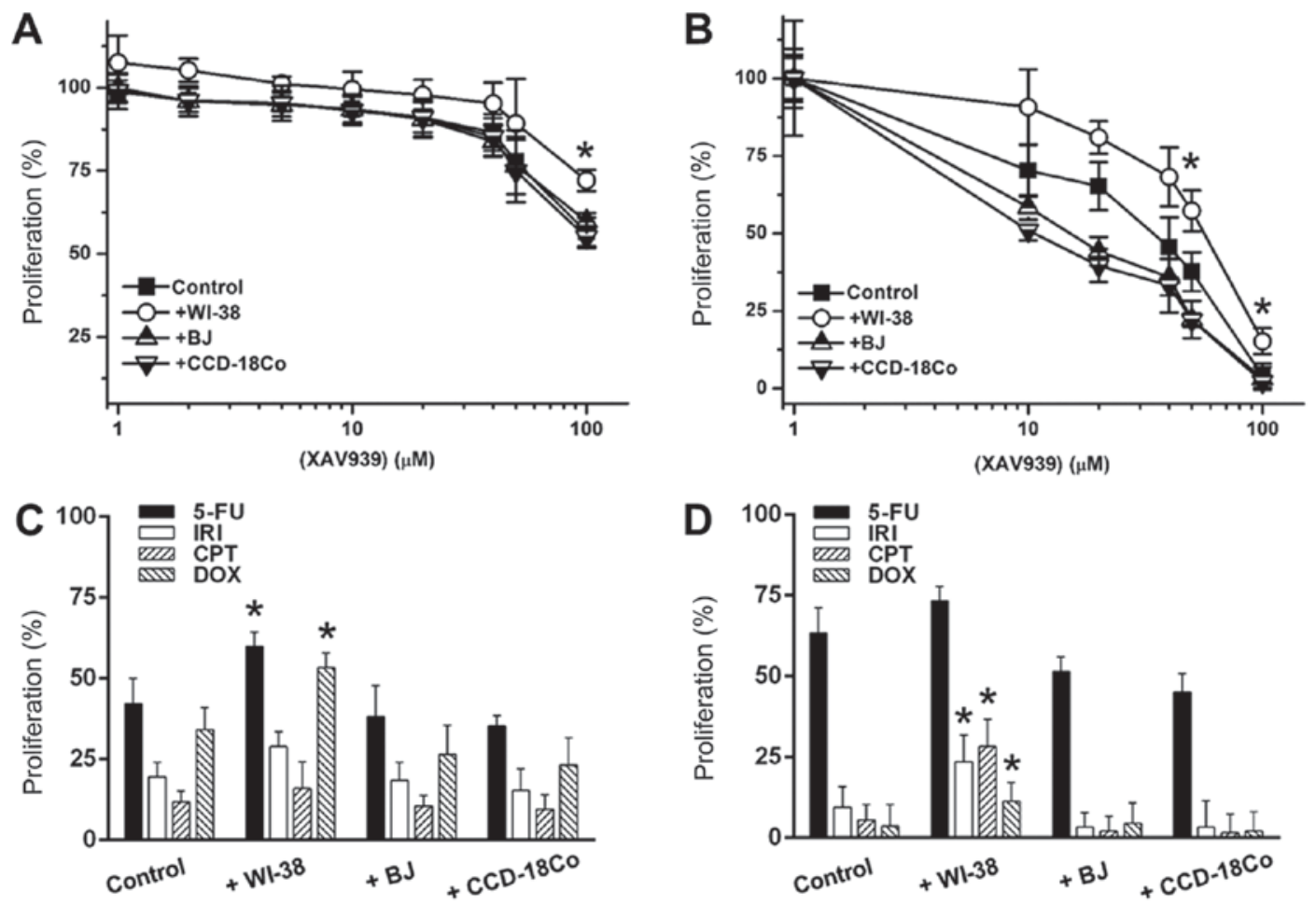

Figure 6. Fibroblast co-culture effect on the response of (A) 2D and (B) anchorage-independent SW480 cells to XAV939. (C and D) The proliferation of (C) 2D and (D) anchorage-independent SW480 cells following the application of anti-cancer drugs. Error bars represent the standard deviation of three replicates. ${ }^{*} \mathrm{P}<0.05$ vs. control. 5-FU, 5-fluorouracil; IRI, irinotecan; CPT, camptothecin; DOX, doxorubicin.
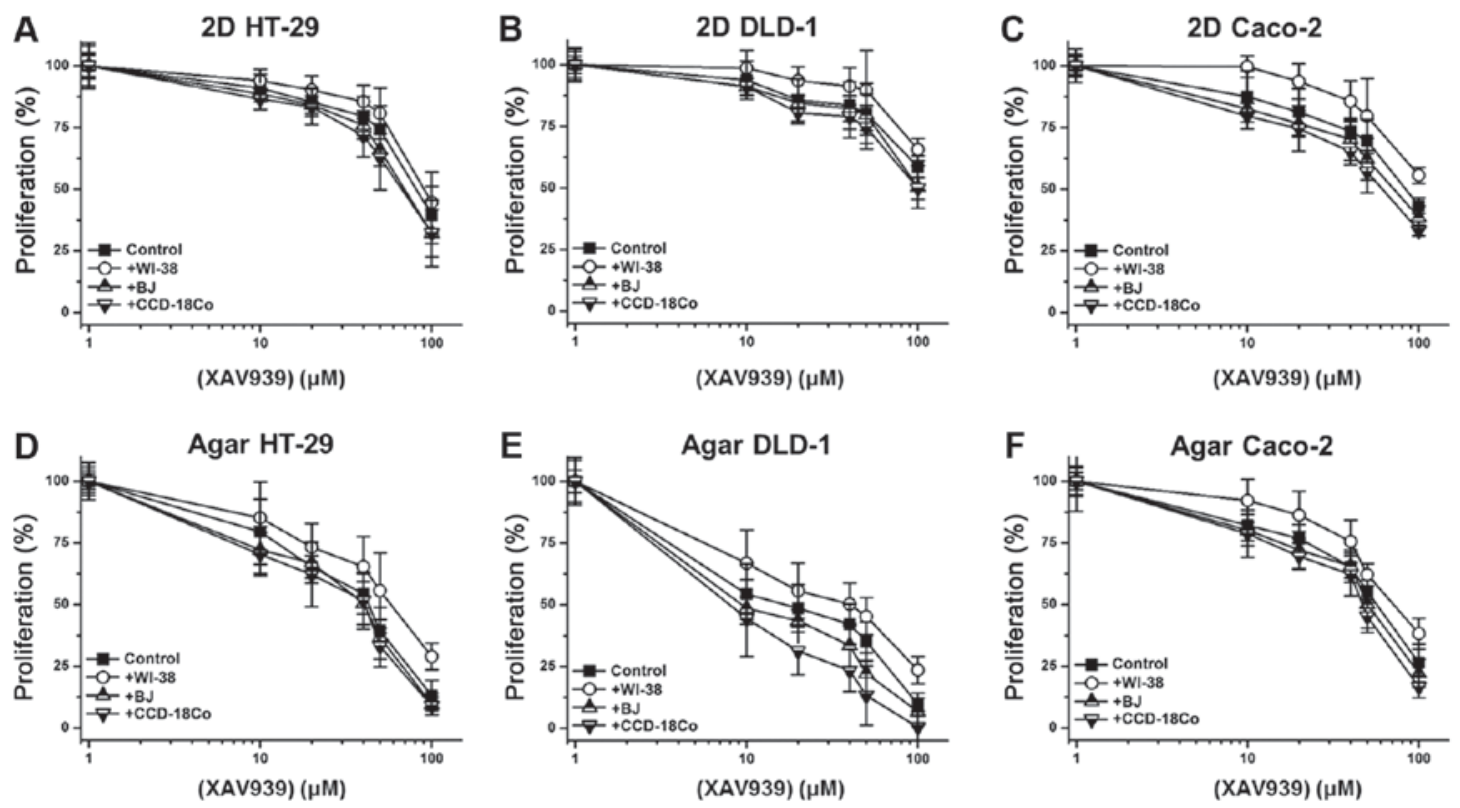

Figure 7. Fibroblast co-culture effect on the response of (A) 2D HT-29, (B) 2D DLD-1, (C) 2D Caco-2, (D) agar HT-29, (E) agar DLD-1 and (F) agar Caco-2 cells to XAV939. Error bars represent the standard deviation of three replicates.

aberrant WNT signaling is recognized as an early event in the development of colorectal cancer (43). BJ and CCD-18Co co-cultures caused $>3 \%$ changes in the response of cancer cells to XAV939, while WI-38 co-culture significantly reduced the XAV939-sensitivity of colon cancer cells. 2D and anchorage independent SW480 cultures exhibited disparate responses to XAV939. Kim et al (44) suggested that this difference can be attributed by differential expression of lactate dehydrogenase
A and gelsolin. The effect of 5-FU, IRI, CPT and DOX on the colon cancer-fibroblast co-culture system was also tested to investigate the relationship between different signaling pathways and cell proliferation in the anchorage independent condition. It has been reported that 5-FU induces mitochondrial apoptosis in colon cancer cells lacking p53, while IRI, CPT and DOX inhibit topoisomerase resulting in DNA damage and apoptosis (45-47). Against these anti-cancer 
agents (5-FU, IRI, CPT and DOX), WI-38 co-culture also reduced the colon cancer cell drug response. By contrast, BJ and CCD-18Co co-cultures increased sensitivity to all 5 drugs tested, further supporting their role as negative regulators of cancer cell proliferation. Similarly, WI-38 conditioned medium enhanced colon cancer cell proliferation while causing little or no decrease in the rate of apoptosis, whereas BJ/CCD-18Co medium reduced colon cancer cell proliferation. However, the mechanism underlying the interaction between colon cancer cells and fibroblasts are not yet fully understood, and our group is currently investigating protein, metabolite and lipid expression patterns associated with colon cancer growth in the presence of fibroblasts. As p53 might play on important role in the cancer microenvironment, we are currently investigating its role in cancer proliferation.

In conclusion, fibroblasts may act as positive or negative regulators of colon cancer cell proliferation depending on the fibroblast cell type. Factors released by different types of non-cancerous fibroblasts may have an impact on cancer cell proliferation, apoptosis and drug responses. Thus, when targeting TME to treat specific cancers, fibroblasts in the tissue should be carefully examined and either positive/negative modulation of the TME should be applied depending on the prevailing fibroblast-mediated regulation of cancer.

\section{Acknowledgements}

The authors would like to thank Dr. Sang Dal Rhee, Dr. Ki Young Kim and Dr. Hyejin Nam (Korea Research Institute of Chemical Technology, Daejeon, Republic of Korea) for their helpful discussions. The authors would also like to thank Mr. Jonghyum Kim and Professor Tae-Young Kim (both of Gwangju Institute of Science and Technology, Gwangju, Republic of Korea) for assisting with the acquisition of data.

\section{Funding}

The present study was financially supported by the R\&D Convergence Program of National Research Council of Science \& Technology (grant no. CAP-15-10-KRICT), and the Korea Research Institute of Chemical Technology core project (grant no. KK1703-F00).

\section{Availability of data and materials}

The datasets used during the present study are available from the corresponding author upon reasonable request.

\section{Authors' contributions}

BK, DuK and KRK conceived and designed the study. BK, $\mathrm{HJ}, \mathrm{DaK}$ and DuK performed the experiments. BK wrote the manuscript. BK, HJ, DaK, DuK and KRK reviewed and edited the manuscript. All authors read and approved the manuscript and agree to be accountable for all aspects of the research in ensuring that the accuracy or integrity of any part of the work are appropriately investigated and resolved. All authors have given approval to the final version of the manuscript.

\section{Ethics approval and consent to participate}

Not applicable.

\section{Patient consent for publication}

Not applicable.

\section{Competing interest}

The authors declare that they have no competing interests.

\section{References}

1. Siegel RL, Miller KD and Jemal A: Cancer statistics, 2018. CA Cancer J Clin 68: 7-30, 2018.

2. Jung KW, Won YJ, Kong HJ, Oh CM, Cho H, Lee DH and Lee KH: Cancer statistics in Korea: Incidence, mortality, survival, and prevalence in 2012. Cancer Res Treat 47: 127-141, 2015.

3. Wang D, Rai B, Qi F, Liu T, Wang J, Wang X and Ma B: Influence of the Twist gene on the invasion and metastasis of colon cancer. Oncol Rep 39: 31-44, 2018.

4. Duan L, Deng L, Wang D, Ma S, Li C and Zhao D: Treatment mechanism of matrine in combination with irinotecan for colon cancer. Oncol Lett 14: 2300-2304, 2017.

5. Li K, Guo J, Wu Y, Jin D, Jiang H, Liu C and Qin C: Suppression of YAP by DDP disrupts colon tumor progression. Oncol Rep 39: 2114-2126, 2018.

6. De Roock W, De Vriendt V, Normanno N, Ciardiello F and Tejpar S: KRAS, BRAF, PIK3CA, and PTEN mutations: Implications for targeted therapies in metastatic colorectal cancer. Lancet Oncol 12: 594-603, 2011.

7. Moriarity A, O'Sullivan J, Kennedy J, Mehigan B and McCormick P: Current targeted therapies in the treatment of advanced colorectal cancer: A review. Ther Adv Med Oncol 8: 276-293, 2016.

8. Hagan S, Orr MC and Doyle B: Targeted therapies in colorectal cancer-an integrative view by PPPM. EPMA J 4: 3, 2013.

9. Heinemann V, Douillard JY, Ducreux M and Peeters M: Targeted therapy in metastatic colorectal cancer-an example of personalized medicine in action. Cancer Treat Rev 39: 592-601, 2013.

10. Hwang TJ, Carpenter D, Lauffenburger JC, Wang B, Franklin JM and Kesselheim AS: Failure of investigational drugs in late-stage clinical development and publication of trial results. JAMA Intern Med 176: 1826-1833, 2016.

11. Hay M, Thomas DW, Craighead JL, Economides C and Rosenthal J: Clinical development success rates for investigational drugs. Nat Biotechnol 32: 40-51, 2014.

12. Sun Y: Tumor microenvironment and cancer therapy resistance. Cancer Lett 380: 205-215, 2016.

13. Fang $\mathrm{H}$ and Declerck YA: Targeting the tumor microenvironment: From understanding pathways to effective clinical trials. Cancer Res 73: 4965-4977, 2013.

14. Chen F, Zhuang X, Lin L, Yu P, Wang Y, Shi Y, Hu G and Sun Y: New horizons in tumor microenvironment biology: Challenges and opportunities. BMC Med 13: 45, 2015.

15. Peddareddigari VG, Wang D and Dubois RN: The Tumor microenvironment in colorectal carcinogenesis. Cancer Microenviron 3: 149-166, 2010.

16. Zhou W, Xu G, Wang Y, Xu Z, Liu X, Xu X, Ren G and Tian K: Oxidative stress induced autophagy in cancer associated fibroblast enhances proliferation and metabolism of colorectal cancer cells. Cell Cycle 16: 73-81, 2017.

17. O'Toole A, Michielsen AJ, Nolan B, Tosetto M, Sheahan K, Mulcahy HE, Winter DC, Hyland JM, O'Connell PR, Fennelly D, et al: Tumour microenvironment of both early- and late-stage colorectal cancer is equally immunosuppressive. Br J Cancer 111: 927-932, 2014.

18. Jacobs J, Smits E, Lardon F, Pauwels P and Deschoolmeester V: Immune checkpoint modulation in colorectal cancer: What's new and what to expect. J Immunol Res 2015: 158038, 2015.

19. Colangelo T, Polcaro G, Muccillo L, D'Agostino G, Rosato V, Ziccardi P, Lupo A, Mazzoccoli G, Sabatino L and Colantuoni V: Friend or foe?: The tumour microenvironment dilemma in colorectal cancer. Biochim Biophys Acta Rev Cancer 1867: 1-18, 2017. 
20. Cammarota R, Bertolini V, Pennesi G, Bucci EO, Gottardi O, Garlanda C, Laghi L, Barberis MC, Sessa F, Noonan DM and Albini A: The tumor microenvironment of colorectal cancer: Stromal TLR-4 expression as a potential prognostic marker. J Transl Med 8: 112, 2010.

21. Marsh T, Pietras K and McAllister SS: Fibroblasts as architects of cancer pathogenesis. Biochim Biophys Acta 1832: 1070-1078, 2013.

22. Kalluri R: The biology and function of fibroblasts in cancer. Nat Rev Cancer 16: 582-598, 2016.

23. Kalluri R and Zeisberg M: Fibroblasts in cancer. Nat Rev Cancer 6: 392-401, 2006.

24. Lotti F, Jarrar AM, Pai RK, Hitomi M, Lathia J, Mace A, Gantt GA Jr, Sukhdeo K, DeVecchio J, Vasanji A, et al: Chemotherapy activates cancer-associated fibroblasts to maintain colorectal cancer-initiating cells by IL-17A. J Exp Med 210: 2851-2872, 2013

25. Ostman A and Augsten M: Cancer-associated fibroblasts and tumor growth-bystanders turning into key players. Curr Opin Genet Dev 19: 67-73, 2009.

26. Iacopino F, Angelucci C and Sica G: Interactions between normal human fibroblasts and human prostate cancer cells in a co-culture system. Anticancer Res 32: 1579-1588, 2012.

27. Tao L, Huang G, Song H, Chen Y and Chen L: Cancer associated fibroblasts: An essential role in the tumor microenvironment. Oncol Lett 14: 2611-2620, 2017.

28. Shiga K, Hara M, Nagasaki T, Sato T, Takahashi $\mathrm{H}$ and Takeyama $\mathrm{H}$ : Cancer-associated fibroblasts: Their characteristics and their roles in tumor growth. Cancers (Basel) 7: 2443-2458, 2015.

29. Zabransky DJ, Yankasakas CL, Cochran RL, Wong HY, Croessmann S, Chu D, Kavuri SM, Red Brewer M, Rosen DM, Dalton WB, et al: HER2 missense mutations have distinct effects on oncogenic signaling and migration. Proc Natl Acad Sci USA 112: E6205-E6214, 2015.

30. Hwang RF, Moore T, Arumugam T, Ramachandran V, Amos KD, Rivera A, Ji B, Evans DB and Logsdon CD: Cancer-associated stromal fibroblasts promote pancreatic tumor progression. Cancer Res 68: 918-926, 2008.

31. Liao D, Luo Y, Markowitz D, Xiang R and Reisfeld RA: Cancer associated fibroblasts promote tumor growth and metastasis by modulating the tumor immune microenvironment in a 4T1 murine breast cancer model. PLoS One 4: e7965, 2009.

32. Cheng HH, Chu LY, Chiang LY, Chen HL, Kuo CC and Wu KK Inhibition of cancer cell epithelial mesenchymal transition by normal fibroblasts via production of 5-methoxytryptophan. Oncotarget 7: 31243-31256, 2016.

33. de Toledo M, Anguille C, Roger L, Roux P and Gadea G: Cooperative anti-invasive effect of $\mathrm{Cdc} 42 / \mathrm{Rac1}$ activation and ROCK inhibition in SW620 colorectal cancer cells with elevated blebbing activity. PLoS One 7: e48344, 2012

34. Majety M, Pradel LP, Gies M and Ries CH: Fibroblast influence survival and therapeutic response in a $3 \mathrm{~d}$ Co-culture model. PLoS One 10: e0127948, 2015.
35. Yamada T, Matsumoto K, Wang W, Li Q, Nishioka Y, Sekido Y, Sone S and Yano S: Hepatocyte growth factor reduces susceptibility to an irreversible epidermal growth factor receptor inhibitor in EGFR-T790M mutant lung cancer. Clin Cancer Res 16: 174-183. 2010

36. Villeneueve PJ and Sundaresan RS: Surgical management of colorectal lung metastasis. Clin Colon Rectal Surg 22: 233-241, 2009.

37. Nesseris I, Tsamakis C, Gregoriou S, Ditsos I, Christofidou E and Rigopoulos D: Cutaneous metastasis of colon adenocarcinoma: Case report and review of the literature. An Bras Dermatol 88 (6 Suppl 1): S56-S58, 2013.

38. Liu Y, Zhang F, Zhang XF, Qi LS, Yang L, Guo H and Zhang N: Expression of nucleophosmin/NPM1 correlates with migration and invasiveness of colon cancer cells. J Biomed Sci 19: 53, 2012.

39. Mouradov D, Sloggett C, Jorissen RN, Love CG, Li S, Burgess AW, Arango D, Strausberg RL, Buchanan D, Wormald S, et al: Colorectal cancer cell lines are representative models of the main molecular subtypes of primary cancer. Cancer Res 74: 3238-3247, 2014

40. Labi V and Erlacher M: How cell death shapes cancer. Cell Death Dis 6: e1675, 2015

41. Wong RS: Apoptosis in cancer: From pathogenesis to treatment. J Exp Clin Cancer Res 30: 87, 2011.

42. Trédan O, Galmarini CM, Patel K and Tannock IF: Drug Resistance and the solid tumor microenvironment. J Natl Cancer Inst 99: 1441-1454, 2007.

43. Segditsas S and Tomlinson I: Colorectal cancer and genetic alterations in the Wnt pathway. Oncogene 25: 7531-7537, 2006.

44. Kim YE, Jeon HJ, Kim D, Lee SY, Kim KY, Hong J, Maeng PJ, Kim KR and Kang D: Quantitative proteomic analysis of $2 \mathrm{D}$ and 3D cultured colorectal cancer cells: Profiling of tankyrase inhibitor xav939-induced proteome. Sci Rep 8: 13255. 2018

45. Pagliara V, Saide A, Mitidieri E, d'Emmanuele di Villa Bianca R, Sorrentino R, Russo G and Russo A: 5-FU targets rpL3 to induce mitochondrial apoptosis via cystathionine- $\beta$-synthase in colon cancer cells lacking p53. Oncotarget 7: 50333-50348, 2016.

46. Fuchs C, Mitchell EP and Hoff PM: Irinotecan in the treatment of colorectal cancer. Cancer Treat Rev 32: 491-503, 2006.

47. Weinländer G, Kornek G, Raderer M, Hejna M, Tetzner C and Scheithauer W: Treatment of advanced colorectal cancer with doxorubicin combined with two potential multidrug-resistance-reversing agents: High-dose oral tamoxifen and dexverapamil. J Cancer Res Clin Oncol 123: 452-455, 1997.

(i) $(-)$ This work is licensed under a Creative Commons Attribution-NonCommercial-NoDerivatives 4.0 International (CC BY-NC-ND 4.0) License. 